\title{
Determination of right ventricular volume and function using multiple axially rotated MRI slices
}

\author{
Meriam Aneq Åström, Eva Nylander, Tino Ebbers and Jan Engvall
}

\section{Linköping University Post Print}

N.B.: When citing this work, cite the original article.

This is the authors' version of the following article:

Meriam Aneq Åström, Eva Nylander, Tino Ebbers and Jan Engvall, Determination of right ventricular volume and function using multiple axially rotated MRI slices, 2011, CLINICAL PHYSIOLOGY AND FUNCTIONAL IMAGING, (31), 3, 233-239.

which has been published in final form at:

http://dx.doi.org/10.1111/j.1475-097X.2011.01006.x

Copyright: Blackwell Publishing Ltd http://eu.wiley.com/WileyCDA/Brand/id-35.html

Postprint available at: Linköping University Electronic Press http://urn.kb.se/resolve?urn=urn:nbn:se:liu:diva-67837 


\section{Determination of right ventricular volume and function using multiple axially rotated MRI slices.}

Meriam Åström Aneq ${ }^{1-3^{*}}$, Eva Nylander ${ }^{1-3}$, Tino Ebbers ${ }^{3-5,}$ Jan Engvall $^{1-3}$

${ }^{1}$ Clinical physiology Department, Heart centre, University Hospital, Linköping, Sweden

${ }^{2}$ Department of Medicine and Health Sciences/Cardiovascular medicine/Clinical Physiology,

Linkoping University, Linkoping, Sweden

${ }^{3}$ Centre for Medical Image Science and Visualization, Linköping University, Linköping,

Sweden

${ }^{4}$ Department of Medicine and Health Sciences/Cardiovascular medicine/Physiology,

Linkoping University, Linkoping, Sweden

${ }^{5}$ Division of Applied Thermodynamics and Fluid Mechanics, Dept. of Management and

Engineering, Linköping University, Linköping Sweden

* Corresponding author

Email addresses:

MÅA: meriam.astrom.aneq@lio.se

JE : jan.engvall@lio.se

EN : eva.nylander@lio.se

TE : tino.ebbers@liu.se 


\section{Abstract}

\section{Background}

The conventional MRI method for right ventricular (RV) volume and motion, using short-axis (SA) orientation, is limited by RV anatomy and shape. We suggest an orientation based on six slices rotated around the long axis of the RV, rotated long axis (RLA).

\section{Material and method}

Three phantoms were investigated in SA and RLA using cine balanced steady state free precession MRI. Volumes were calculated based on segmentation and checked against true volumes.

In 23 healthy male volunteers we used six long axis planes from the middle of the tricuspid valve to the RV apex, rotated in 30 degree increments. For comparison, short axis slices were acquired. Imaging parameters were identical in both acquisitions.

\section{Results}

RV end-diastolic, end-systolic and stroke volumes determined in the RLA 179.1 $\pm 29.3 ; 80.1$ $\pm 17.1 ; 99.3 \pm 16.9 \mathrm{ml}$ and in the SA were $174.0 \pm 21.1 ; 78.8 \pm 13.6 ; 95.3 \pm 14.5 \mathrm{ml}$ with $\mathrm{p}$ values for the difference from 0.17 to 0.64 (ns). Inter-observer variability ranged between $3.2 \%$ and $6.6 \%$, intra-observer variability between $2.8 \%$ and $6.8 \%$. In SA views, consensus for the definition of the basal slice was necessary in $39 \%$ of the volunteers for whom the average volume change was $20 \%$ in end-systolic and $10 \%$ in end-diastolic volume.

\section{Conclusions}

The RLA method results in better visualisation and definition of the RV inflow, outflow and apex. Accurate measurement of RV volumes for diagnosis and follow up of cardiac diseases is enhanced by the RLA orientation, even though additional acquisition time is required. 


\section{Introduction}

Assessment of left ventricular function (LV) has always been central within cardiac investigations, while right ventricular (RV) volume and motion have, at times, been overlooked. Right ventricular function may be impaired in many different disease states, such as congenital heart disease, pulmonary hypertension, coronary heart disease, as well as in inherited cardiomyopathies. For a comprehensive cardiac assessment, RV function also needs careful attention. The complex shape and orientation of the right ventricle, being more or less "wrapped around" the left ventricle (Torrent-Guasp et al., 2001), is a challenging factor in the visualization and assessment of RV function. Magnetic resonance imaging (MRI) has offered new opportunities in the study of this cardiac chamber. Compared with other modalities such as cardiac computerized tomography (CCT) and EKG-gated blood pool scintigraphy, MRI has a higher temporal resolution (Koch et al., 2005; Lembcke et al., 2005) and avoids the need for ionizing radiation. Furthermore, MRI has the ability to visualize all parts of the RV. This is rarely possible with echocardiography, which is otherwise the primary imaging modality for structural and functional cardiac investigations. In the routine assessment of RV function, multiple slices are acquired in a cross-sectional, short axis (SA) orientation, covering both the RV and the LV (Sechtem et al., 1987; Higgins et al., 1988). However this evaluation of the RV is complicated by its asymmetric form. True transverse images of the $\mathrm{RV}$ are difficult to acquire, since the long axis is often curved between apex and base. Another limitation is the large longitudinal excursion of the tricuspid annulus which complicates the definition of the basal slice of the RV during the cardiac cycle. This may lead to large uncertainty in RV assessment, since the volume contribution of the basal slice is substantial due to the extreme cone shape of the RV. 
We hypothesized that an acquisition method based on six slices rotated around the long axis of the right ventricle would facilitate the visualisation and definition of the RV inflow, outflow and apex during the various phases of the cardiac cycle. The feasibility of this acquisition was assessed by validation in phantom studies and in healthy volunteers, comparing the rotated long axis (RLA) orientation with the traditional acquisition of SA images, and calculating intra- and inter-observer variability of calculated volumes.

\section{Material and Methods}

\section{Image acquisition}

\section{Phantoms}

A phantom study was performed to investigate the accuracy of the volume estimation for different geometries using both acquisition strategies: slices acquired in the RLA and SA direction. Since the shape of the right heart is crescentic, coned, and varying over the cardiac cycle, we used a banana, a pear and a bottle to investigate the ability to calculate the volume for different geometries. MR exams were performed using a Philips 1.5T Achieva scanner (Philips Healthcare, Best, the Netherlands). We acquired images with a k-space segmented balanced steady state free precession (b-SSFP) sequence. For all phantoms, six RLA slices were acquired, and sufficient SA slices to cover the complete phantom. Imaging parameters, TE $10 \mathrm{~ms}$, flip angle $60^{\circ}$, slice thickness $8 \mathrm{~mm}$ and pixel size $1.25 \times 1.25 \mathrm{~mm}^{2}$, were identical for both the RLA and SA acquisitions. True phantom volumes were measured by immersing the objects in a beaker of water.

\section{Human volunteers}


We studied 23 healthy male volunteers, mean age 39 years, (range 22-64). They were recruited by advertisements on the university web page and among medical students. Exclusion criteria were past medical history, ongoing medication, arrhythmia or elevated blood pressure. All volunteers underwent a cardiac exercise test to confirm the absence of heart disease. The mean body mass index was 23.1 (range 18.8-28.1). The systolic blood pressure ranged between 102 to 140 , mean $122 \mathrm{mmHg}$, with diastolic ranging between 60 to 85, mean $71 \mathrm{mmHg}$. The study was approved by the Regional Ethics Review Board. Written informed consent was obtained from all subjects.

The volunteers were investigated with the $1.5 \mathrm{~T}$ MRI system, using a five-element cardiac synergy surface coil. Retrospectively ECG gated MR images were obtained during repeated breath-holds. Cine-MR was performed with a k-space segmented b-SSFP sequence. Three long axis planes of the left ventricle (2- and 4- chamber views as well as the apical long-axis view) were obtained for orientation. The SA was planned on the LV, covering the entire heart with on average of 19 (range 17-25) short-axis slices. Six long axis planes were acquired, rotated in $30^{\circ}$ increment around the right ventricular long axis which was drawn from the middle of the tricuspid valve to the right ventricular apex. Acquisition time for every long axis slice was 12 heart beats, while every short axis slice took 6 heart beats to acquire due to the use of a SENSE factor 2. Otherwise, imaging parameters, echo time (TE) $1.5 \mathrm{ms,}$ repetition time (TR) $3.0 \mathrm{~ms}$, flip angle $60^{\circ}$, slice thickness $8 \mathrm{~mm}$ and pixel size $1.25 \times 1.25$ $\mathrm{mm}^{2}$, were identical for both RLA and SA acquisition. Thirty cardiac phases were reconstructed for all slices. The order of the sequence of axial and short-axis acquisition was assigned randomly for each patient. Since each slice required a separate breath hold, 6 slices were considered a suitable compromise between the accuracy of the calculation and the time needed for the acquisition. 
In addition, two-dimensional cine through-plane PC-MRI velocity data were acquired in a plane perpendicular to the main flow direction in the ascending aorta (AoA), just downstream from the aortic valve (AoV). Imaging parameters included a velocity encoding range (VENC) of $200 \mathrm{~cm} / \mathrm{s}$, TE of $3 \mathrm{~ms}$, TR $5 \mathrm{~ms}$, flip angle $15^{\circ}$, slice thickness $8 \mathrm{~mm}$, and a pixel size of 1.6 $\mathrm{x} 1.6 \mathrm{~mm}^{2}$. Three lines of $\mathrm{k}$-space were acquired per heart beat, resulting in a temporal resolution of $30 \mathrm{~ms}$. All scans were performed by a single operator.

\section{Image analysis}

Phantom volumes from still images, as well as cardiac cine images were evaluated using the freely available analysis software Segment (v1.702, http://segment.heiberg.se). The six axially rotated slices of the right ventricle were presented in cine. Volume measurements were performed on right ventricular rotated axial images in addition to left and right ventricular short axis acquisitions. For the RLA-presentation of the right ventricle, the software "Segment" calculates the volume of a polygon delimited by the boundaries of the six rotated slices, in diastole as well as in systole. Each slice will contribute two wedges of 30o central angle opposite to each other and united by the centerline of the polygon. Segment sweeps the boundary from one edge to the other of the wedges in small incremental steps. A description and validation of the Segment software has been recently published (Heiberg et al., 2010). LV and RV volumes were also calculated as summed stacks of slices from the short axis view. The endocardial borders of the LV and RV were proposed automatically by the software. The operator was allowed to manually adjust the endocardial LV and RV contours at end diastole (first cine phase of the retrospectively R-wave gated stack) and at end systole, defined visually as the image phase with the smallest cavity areas guided by the valves opening and closing movements. For both ventricles, trabeculae and papillary muscles were excluded from the delineated ventricular wall. The LV and RV end-diastolic (EDV) and end-systolic 
volumes (ESV) were calculated and, based on them, stroke volume (SV) and ejection fraction (EF) were calculated. From the rotated slices, a polyhedral structure was determined from the segmentation and its volume calculated (figures 1 and 2). Two observers measured all the data independently. For the short axis datasets, a consensus was reached before segmentation on which basal slice to include. The volume of added, as well as of deleted slices was analyzed independently and the stroke volume recalculated. Consensus was not necessary for the left ventricle, when using the principle of requiring at least half of the periphery of the ventricular slice to be present in each time frame. For the subsequent analysis of the performance of both methods, the mean value from the two observers was used. To determine the intra- observer variability, one observer repeated measurements of RV volumes at least three months later. In the second analysis, the operator was blinded to the results of the first measurements. Aortic flow volume (stroke volume) was calculated with View Forum R6.3 (Philips Healthcare, Best, the Netherlands) as the mean velocity time flow area and was measured on phase contrast images of the ascending aorta, just above the coronary ostia.

\section{Statistics}

All values are presented as mean $\pm \mathrm{SD}$. The statistical significance of differences was calculated using the paired Student's t-test. Significance was based on p values of less than 0.05. To examine the agreement of the two views, the method of Bland and Altman(BLAND, 1983) was used. Agreement was determined as the mean difference between the two views, with limits determined from the mean value \pm 1.96 standard deviations. The intra- and interobserver variabilities were expressed as the coefficient of variability, which is the standard deviation of the differences divided by the mean of the measurements and expressed as a 
percentage. Statistical analyses were performed using Statistica 8.0 (Statsoft Inc, Tulsa, Oklahoma, USA).
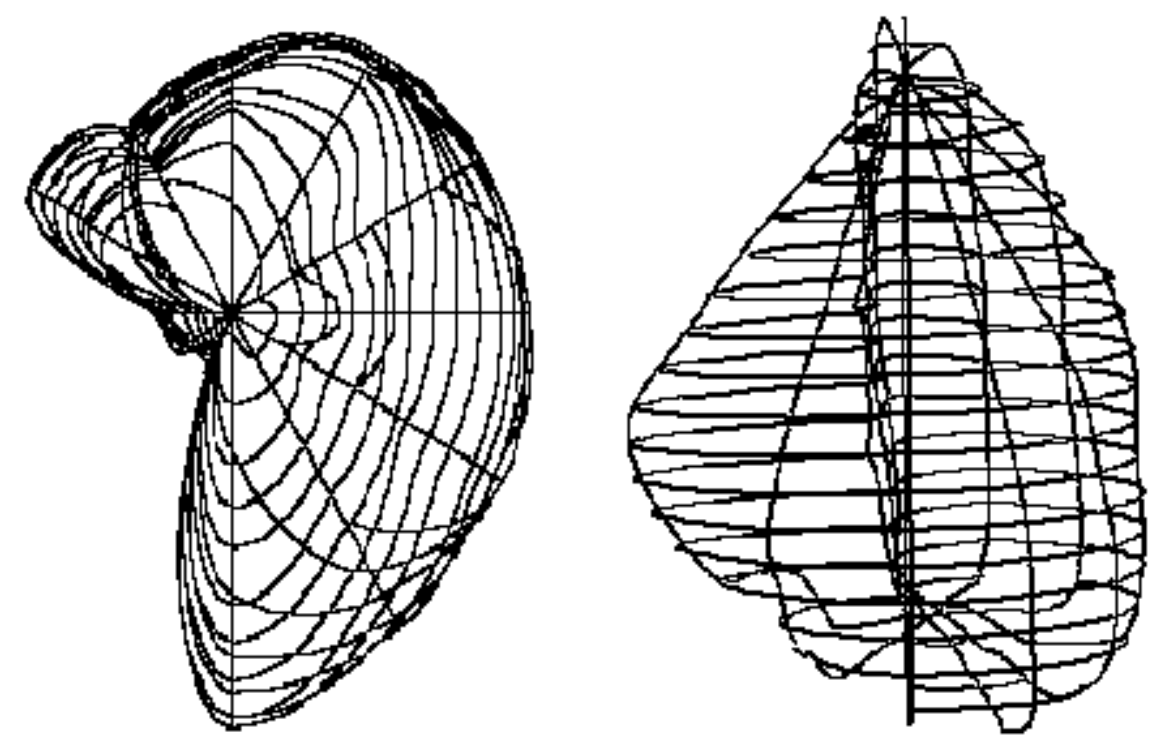

Figure 1: $3 D$ visualization of the six slices rotated around the long axis of the right ventricular chamber.

\section{Results}

Phantom volume calculations performed from both the SA and RLA acquisitions agreed closely with each other and with the volume of displaced water. For the pear and the bottle, the difference compared with the real volume was only $1 \mathrm{ml}(0.5 \%)$ while for the banana, it was $3 \mathrm{ml}(2.3 \%)$ smaller for the short axis calculation and $3 \mathrm{ml}$ larger for the long axis calculation. In the volunteer studies, image quality was satisfactory and a successful calculation of LV and RV volumes was possible on all recordings. Mean heart rate during SA acquisition was $69.6 \pm 12.2$ and during RLA $66.9 \pm 9.9$, with a mean difference of $2.6 \pm 6.6$ bpm. After consensus between the two observers (SA view), the EDV, ESV, SV and EF for the right ventricle did not differ between the RLA and SA views measure (after consensus about the basal slice) with p-values in excess of 0.05 (Table 1, figure 3). 

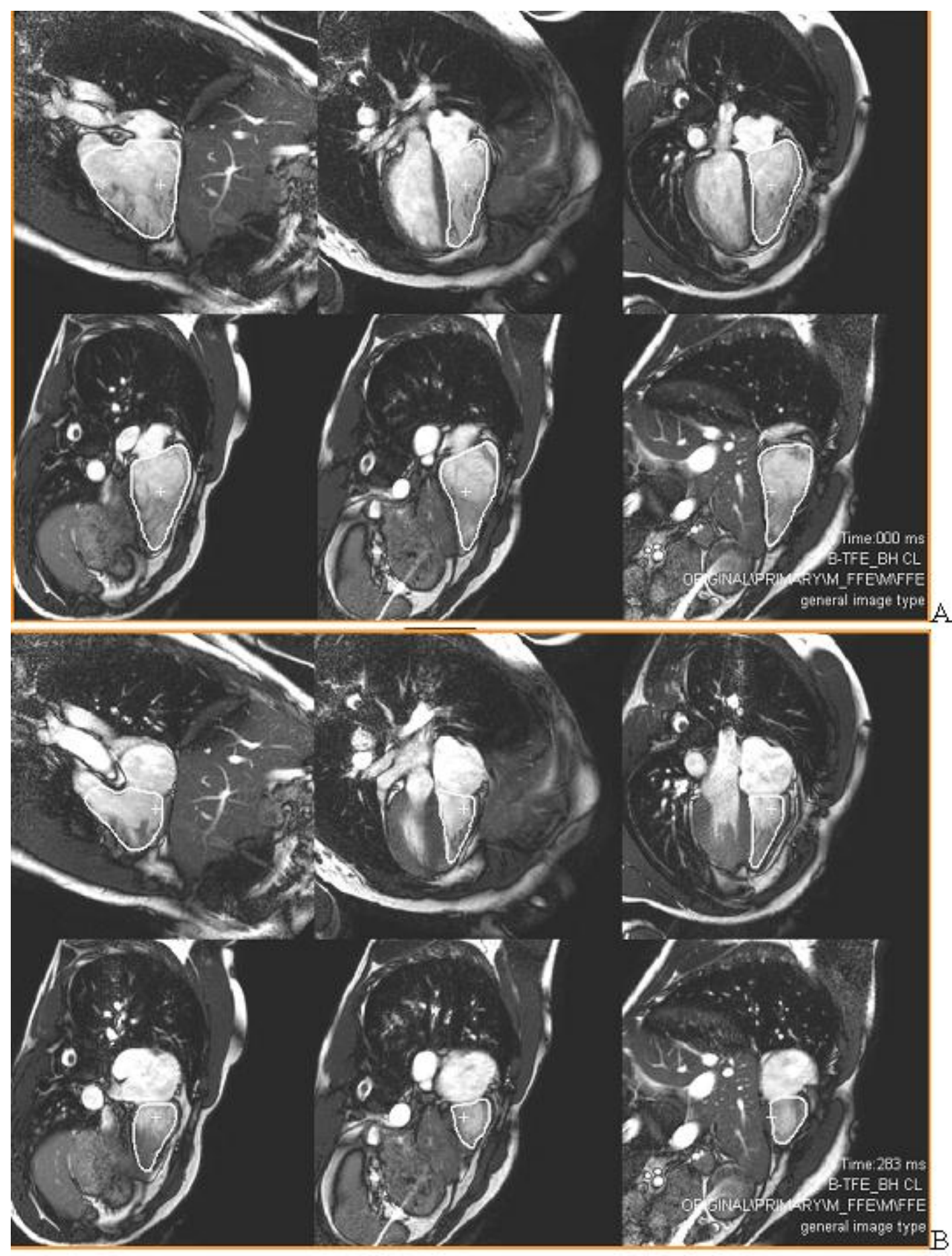

Figure 2 Rotated axial images with endocardial contours defined for the right ventricle in end diastole $(A)$ and in end systole $(B)$.

LV stroke volume was successfully measured with phase contrast MRI in 20 of the 23

volunteers and did not differ from anatomical LV stroke volume calculations, $104.5 \pm 21.3$ vs.

107.8 $\pm 17.3, \mathrm{p}>0.57$ (Table 1) respectively. Three flow studies could not be retrieved from the

PACS for technical reasons. Interobserver coefficients of variability were for the left 
ventricular EDV, ESV, SV and EF 2.46\%, 5.53\%; $2.57 \%$ and $2.12 \%$, respectively. The interand intra-observer variability for the RV volumes and ejection fraction were with the SA orientation $3.2 \%$ to $6.9 \%$ and for the RLA $2.8 \%$ to $6.3 \%$, with a tendency towards a lower CV for stroke volume determined in the RLA compared with the SA (Table 2).

Table 1 Right and left ventricular volumes. Mean values \pm SD of left ventricular (LV) and right ventricular (RV) short axis (SA), RV-rotated long-axis (RLA) dimensions and functions using cine SSFP MRI. Aortic SV from cine phase-contrast MRI.

\begin{tabular}{|c|c|c|c|c|c|}
\hline & \multicolumn{2}{|c|}{ Right ventricle } & \multirow[b]{2}{*}{$\mathrm{P}$} & \multirow[t]{2}{*}{ Left ventricle } & \multirow[t]{2}{*}{ Aortic flow } \\
\hline & RLA & SA & & & \\
\hline $\mathrm{EDV}(\mathrm{ml})$ & $179.1 \pm 29.3$ & $174.0 \pm 21.1$ & 0.21 & $168.4 \pm 24.2$ & \\
\hline ESV (ml) & $80.1 \pm 17.1$ & $78.8 \pm 13.6$ & 0.64 & $60.7 \pm 12.7$ & \\
\hline $\mathrm{SV} \quad(\mathrm{ml})$ & $99.3 \pm 16.9$ & $95.3 \pm 14.5$ & 0.17 & $107.8 \pm 17.3$ & $104.5 \pm 21.3$ \\
\hline $\mathrm{EF}(\%)$ & $55.6 \pm 4.6$ & $54.9 \pm 5.2$ & 0.50 & $63.9 \pm 5.2$ & \\
\hline
\end{tabular}

$\mathrm{EDV}=$ end diastolic volume, $\mathrm{ESV}=$ end systolic volume, $\mathrm{SV}=$ stroke volume, $\mathrm{EF}=$ ejection fraction.

Although the differences between observers before and after consensus were generally small using the SA view, in 8 out of 23 volunteers consensus required the addition or deletion of a basal slice, most often in the calculation of end-systolic volume. This basal slice represented on average a change of $20 \%$ in end-systolic volume and $10 \%$ in end-diastolic volume (Table $3)$. 

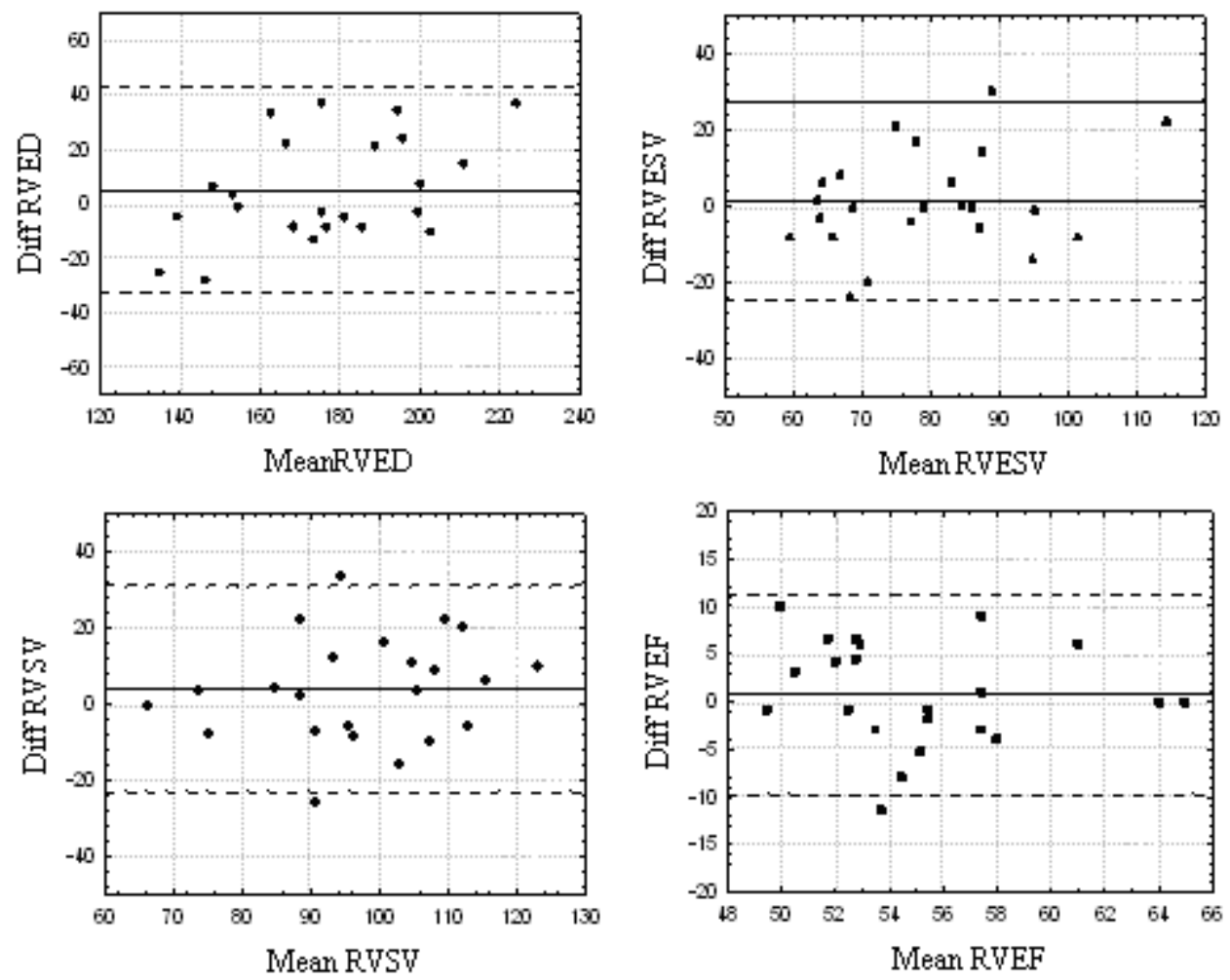

Figure 3 Bland-Altman plot for rotated long axis (RLA) and short axis (SA) acquisition for $R V E D V, R V E S V, R V S V$ and $R V E F$. Solid line = mean difference, dashed lines $=$ mean \pm 1.96 standard deviations.

Table 2: Inter and intra-observer variability (coefficient of variability, \%) for right ventricular volume and function for the rotated long axis and short axis orientations

\begin{tabular}{lllllllll}
\hline \multicolumn{3}{c}{ Rotated long axis } & \multicolumn{5}{c}{ Short axis } \\
\hline & RVEDV & RVESV & RVSV & RVEF & RVEDV & RVESV & RVSV & RVEF \\
Interobserver & $3.3 \%$ & $6.3 \%$ & $3.9 \%$ & $3.3 \%$ & $3.2 \%$ & $6.9 \%$ & $5.9 \%$ & $5.2 \%$ \\
& & & & & & & & \\
Intraobserver & $2.8 \%$ & $5.7 \%$ & $4.8 \%$ & $3.3 \%$ & $3.4 \%$ & $6.6 \%$ & $5.5 \%$ & $6.3 \%$ \\
\hline
\end{tabular}

RVEDV= right ventricular end diastolic volume, RVESV= right ventricular end systolic volume, $\mathrm{RVSV}=$ right ventricular stroke volume, $\mathrm{RVEF}=$ right ventricular ejection fraction. 
Table 3: Effect of deletion (-) or addition (+) of the basal right ventricular slice on the stroke volume in the consensus procedure between observer (obs) 1 and 2 in 8 of 23 healthy volunteers.

Volume differences and stroke volume (SV) are expressed in millilitres (ml) for the individual observer when the number of slices has changed.

RVEDV: Right ventricular end diastolic volume. RVESV: Right ventricular end systolic volume

\begin{tabular}{cclccc}
\hline $\begin{array}{c}\text { Volunteer } \\
\text { number }\end{array}$ & Obs & $\begin{array}{l}\text { Difference in } \\
\text { RVEDV }(\mathrm{ml})\end{array}$ & $\begin{array}{c}\text { Difference in } \\
\text { RVESV }(\mathrm{ml})\end{array}$ & $\begin{array}{l}\text { SV before } \\
\text { consensus }(\mathrm{ml})\end{array}$ & $\begin{array}{c}\text { SV after } \\
\text { consensus }(\mathrm{ml})\end{array}$ \\
\hline 1 & 1 & & -20 & 65 & 85 \\
2 & 1 & & -16 & 69 & 86 \\
2 & 2 & & +12 & 91 & 79 \\
3 & 1 & +18 & & 95 & 113 \\
7 & 2 & & -21 & 82 & 103 \\
9 & 1 & +17 & & 71 & 88 \\
13 & 1 & & +15 & 134 & 119 \\
15 & 1 & & +16 & 93 & 77 \\
17 & 2 & +16 & & 104 & 120 \\
\hline
\end{tabular}

\section{Discussion}

The optimal method for measuring right ventricular volumes is under debate (Watanabe et al., 1986; Koch et al., 2005; Nesser et al., 2006; Williams \& Frenneaux, 2008; Shiota, 2009). The most frequent image orientation uses a series of short axis slices covering both the RV and the LV cavities. This study assesses the use of an alternative orientation for measuring right ventricular volumes with MRI based on six slices equiangularly rotated around an RV long axis between the apex and the tricuspid valve. With this approach, long axis motion will be handled in a better way and motion abnormalities of the wall will be seen more easily. The inter- and intra-observer variability in the measurements showed a tendency to lower values with the RLA approach than with the standard SA orientation (Table 2). In $39 \%$ of volunteers, a large part of the variation in RV volumes in the SA orientation arose from the difficulty in defining the basal slice, especially in end systole. Volumes obtained with the RLA approach corresponded with those from the SA view (after consensus) and were in agreement with previous reports (Alfakih et al., 2003b; Fritz et al., 2005; Tandri et al., 2006). 
It's well known that the challenge using the short axis slices for RV volume measurement has been the definition of the basal slice of the RV and how to handle the RV outflow tract. The latter is out of the plane of the short axis slices, which creates difficulties in the delimitation of the pulmonary valve and the outflow region. In most previous studies, the acquisition protocol for short axis imaging has focused on the left ventricle only. In LV imaging, most slices are parallel to the mitral valve and perpendicular to the interventricular septum (Beygui et al., 2004; Grothues et al., 2004; Kramer et al., 2008; Winter et al., 2008). However, for the right ventricle, this slice positioning does not take into account that the right ventricle more or less wraps around the left ventricle, which result in slices oblique to the right ventricular long axis, especially in the complex anatomy seen in congenital heart disease. Also, the curvature of the right ventricular long axis in combination with the rapid reduction in short axis dimension from the base to the apex creates partial volume effects that make volume measurements imprecise. The definition of the RV basal slice in the short axis views is complicated by the large RV free wall longitudinal excursion (Pattynama et al., 1995; Grothues et al., 2004) which may account for almost $80 \%$ of the RV stroke volume(Carlsson et al., 2007). One property used for differentiating atrial from ventricular slices is the myocardial thickness. However, the RV wall is almost as thin as the atrium and the trabeculations otherwise characteristic of the RV, can be less evident. As the basal slice represents a large contribution of RV volume area, an error in the definition of the inflow part of the RV may result in a significant volume variation.

RV short axis slices may have the potential advantage of being acquired in the same data set as the LV. However, the time needed for recording all RLA slices was only 72 heart beats, which can be compared to 114 heart beats for the average SA study. If enlarged hearts are studied, this difference will be accentuated. Furthermore, the RLA views were acquired without the use of parallel imaging, which, if applied in the future, could speed up acquisition. 
Axial slices perpendicular to the long axis of the body, covering the heart from the pulmonary bifurcation to a level below the diaphragm, have been used as an alternative and have shown superior reproducibility when compared to short axis views in normal individuals and patients with congenital heart disease (Jauhiainen et al., 2002; Lotjonen et al., 2004; Fratz et al., 2009). This orientation, standard for MR imaging, has been advocated as optimal for the visualization of the base, apex and outflow tract of the RV. However, axial imaging can encounter problems with the definition of the outflow tract on the most cranial slice. Another difficulty is the identification of the blood-myocardial boundary especially close to the diaphragm, where the RV wall runs almost parallel to the axial slice (Alfakih et al., 2003a; Fratz et al., 2009). Axial orientation has also been used as localizer for SA orientation and suggested to improve the accuracy of RV volume measurements (Jauhiainen et al., 2002; Strugnell et al., 2005).

We propose that both RLA and the axially oriented slices have the same advantage in bringing out clearly the different parts of right ventricle. Furthermore RLA has the potential to fully account for the right ventricular longitudinal motion. Regardless of selected slice orientation, the delineation of the endocardium and the trabeculae in the thin RV wall is, at times, problematic.

Regional wall motion abnormalities (WMA) are important in the diagnosis of arrhythmogenic RV cardiomyopathy (ARVC), myocardial infarction with RV extension and in some systemic diseases with RV involvement. The rotated long axis orientation allows a better visualization of all the relevant segments, including the inferior and sub tricuspid area. The detection of microaneurysm is an important diagnostic finding in patients suspected of having ARVC and requires an optimal visualization of the perimeter of the RV. In family screening for ARVC, WMA are an important clue to possible early disease and equivocal initial findings necessitate 
follow-up. Objective echocardiographic and MRI measurements have been suggested to complement the visual assessment of the human eye (Kjaergaard et al., 2004; Youssef et al., 2008; Maret et al., 2009). Axially rotated slices could bring out the motion of the RV wall and allow an optimal detection of WMA,. This has to be explored in future studies.

We conclude that acquiring six slices rotated around the long axis of the right ventricle results in a better visualisation and definition of the RV inflow, outflow and apex. The definition of the rotated slices is unambiguous, in contrast to the short axis slices. The only substantial drawback is the additional time required for extra acquisition, as the short axis RV is obtained as a by product of the assessment of the LV. However, this is time well spent in order to obtain an accurate RV diagnosis and follow up of potentially serious cardiac diseases

\section{Limitations}

The short axis and the rotated longitudinal stacks were acquired in sequence with a small time difference. The biological variation of heart rate and stroke volume could possibly have affected the measurements. However, the order of acquisition was random and there were no significant differences in heart rate. The limitation on the use of parallel imaging for rotated acquisition has been removed in presently marketed coils and software. The use of SENSE in future studies could shorten the time needed for such acquisitions.

The number of male volunteers was limited. The full range of biological variation due to age, sex and body size, and to different diseases involving the right ventricle, should be the subject of further investigations. 


\section{Authors' contributions}

MÅA planned the study, investigated all patients, performed measurements and analyses and played a major part in the writing of the manuscript. JE performed measurements and analyses and took part in writing and reviewing the manuscript. EN took part in planning the study, reviewed and discussed the manuscript text. TE took part in the technical support in the MR acquisitions and writing and reviewing the manuscript. All authors have read and approved the final manuscript.

\section{Acknowledgements}

Johan Kihlberg, RN, is gratefully acknowledged for scanning the phantoms and the patients. Einar Heiberg, $\mathrm{PhD}$, provided an updated version of the software Segment.Funding was obtained from the Swedish Heart Lung Foundation, the Faculty of Linköping University, Linköping Heart Centre, the Swedish Society for Research in Sports, the Carldavid Jönsson Foundation, Hjärtfonden and the Stina and Birger Johansson Foundation at Linköping Heart Center as Swedish National Centre for Research in Sports

\section{References}

Alfakih K, Plein S, Bloomer T, Jones T, Ridgway J \& Sivananthan M. (2003a). Comparison of right ventricular volume measurements between axial and short axis orientation using steady-state free precession magnetic resonance imaging. J Magn Reson Imaging 18, 25-32. 
Alfakih K, Plein S, Thiele H, Jones T, Ridgway JP \& Sivananthan MU. (2003b). Normal human left and right ventricular dimensions for MRI as assessed by turbo gradient echo and steady-state free precession imaging sequences. J Magn Reson Imaging 17, 323-329.

Beygui F, Furber A, Delepine S, Helft G, Metzger JP, Geslin P \& Le Jeune JJ. (2004). Routine breath-hold gradient echo MRI-derived right ventricular mass, volumes and function: accuracy, reproducibility and coherence study. Int J Cardiovasc Imaging 20, 509-516.

BLAND DGAaJM. (1983). Measurement in Medicine: the Analysis of Method Comparison Studies. The Statistician 32, 307-317.

Carlsson M, Ugander M, Heiberg E \& Arheden H. (2007). The quantitative relationship between longitudinal and radial function in left, right, and total heart pumping in humans. Am J Physiol Heart Circ Physiol 293, H636-644.

Fratz S, Schuhbaeck A, Buchner C, Busch R, Meierhofer C, Martinoff S, Hess J \& Stern H. (2009). Comparison of accuracy of axial slices versus short-axis slices for measuring ventricular volumes by cardiac magnetic resonance in patients with corrected tetralogy of fallot. Am J Cardiol 103, 1764-1769.

Fritz J, Solaiyappan M, Tandri H, Bomma C, Genc A, Claussen CD, Lima JA \& Bluemke DA. (2005). Right ventricle shape and contraction patterns and relation to magnetic resonance imaging findings. J Comput Assist Tomogr 29, 725-733.

Grothues F, Moon JC, Bellenger NG, Smith GS, Klein HU \& Pennell DJ. (2004). Interstudy reproducibility of right ventricular volumes, function, and mass with cardiovascular magnetic resonance. Am Heart J 147, 218-223.

Heiberg E, Sjogren J, Ugander M, Carlsson M, Engblom H \& Arheden H. (2010). Design and validation of Segment--freely available software for cardiovascular image analysis. BMC Med Imaging 10, 1. 
Higgins CB, Holt W, Pflugfelder P \& Sechtem U. (1988). Functional evaluation of the heart with magnetic resonance imaging. Magn Reson Med 6, 121-139.

Jauhiainen T, Jarvinen VM \& Hekali PE. (2002). Evaluation of methods for MR imaging of human right ventricular heart volumes and mass. Acta Radiol 43, 587-592.

Kjaergaard J, Sogaard P \& Hassager C. (2004). Right ventricular strain in pulmonary embolism by Doppler tissue echocardiography. J Am Soc Echocardiogr 17, 12101212.

Koch K, Oellig F, Oberholzer K, Bender P, Kunz P, Mildenberger P, Hake U, Kreitner KF \& Thelen M. (2005). Assessment of right ventricular function by 16-detector-row CT: comparison with magnetic resonance imaging. Eur Radiol 15, 312-318.

Kramer CM, Barkhausen J, Flamm SD, Kim RJ \& Nagel E. (2008). Standardized cardiovascular magnetic resonance imaging (CMR) protocols, society for cardiovascular magnetic resonance: board of trustees task force on standardized protocols. J Cardiovasc Magn Reson 10, 35.

Lembcke A, Dohmen PM, Dewey M, Klessen C, Elgeti T, Hermann KG, Konertz WF, Hamm B \& Kivelitz DE. (2005). Multislice computed tomography for preoperative evaluation of right ventricular volumes and function: comparison with magnetic resonance imaging. Ann Thorac Surg 79, 1344-1351.

Lotjonen J, Kivisto S, Koikkalainen J, Smutek D \& Lauerma K. (2004). Statistical shape model of atria, ventricles and epicardium from short- and long-axis MR images. Med Image Anal 8, 371-386.

Maret E, Todt T, Brudin L, Nylander E, Swahn E, Ohlsson JL \& Engvall JE. (2009). Functional measurements based on feature tracking of cine magnetic resonance images identify left ventricular segments with myocardial scar. Cardiovasc Ultrasound 7, 53. 
Nesser HJ, Tkalec W, Patel AR, Masani ND, Niel J, Markt B \& Pandian NG. (2006). Quantitation of right ventricular volumes and ejection fraction by three-dimensional echocardiography in patients: comparison with magnetic resonance imaging and radionuclide ventriculography. Echocardiography 23, 666-680.

Pattynama PM, Lamb HJ, Van der Velde EA, Van der Geest RJ, Van der Wall EE \& De Roos A. (1995). Reproducibility of MRI-derived measurements of right ventricular volumes and myocardial mass. Magn Reson Imaging 13, 53-63.

Sechtem U, Pflugfelder PW, Gould RG, Cassidy MM \& Higgins CB. (1987).

Measurement of right and left ventricular volumes in healthy individuals with cine MR imaging. Radiology 163, 697-702.

Shiota T. (2009). 3D echocardiography: evaluation of the right ventricle. Curr Opin Cardiol 24, 410-414.

Strugnell WE, Slaughter RE, Riley RA, Trotter AJ \& Bartlett H. (2005). Modified RV short axis series--a new method for cardiac MRI measurement of right ventricular volumes. J Cardiovasc Magn Reson 7, 769-774.

Tandri H, Daya SK, Nasir K, Bomma C, Lima JA, Calkins H \& Bluemke DA. (2006). Normal reference values for the adult right ventricle by magnetic resonance imaging. Am J Cardiol 98, 1660-1664.

Torrent-Guasp F, Buckberg GD, Clemente C, Cox JL, Coghlan HC \& Gharib M. (2001). The structure and function of the helical heart and its buttress wrapping. I. The normal macroscopic structure of the heart. Semin Thorac Cardiovasc Surg 13, 301319.

Watanabe M, Hosoda Y \& Longmore DB. (1986). [Left and right ventricular dimensions and functions measured by ECG-gated NMR cardiac imaging]. J Cardiogr 16, 343-352. 
Williams L \& Frenneaux M. (2008). Assessment of right ventricular function. Heart 94, 404-405.

Winter MM, Bernink FJ, Groenink M, Bouma BJ, van Dijk AP, Helbing WA, Tijssen JG \& Mulder BJ. (2008). Evaluating the systemic right ventricle by CMR: the importance of consistent and reproducible delineation of the cavity. $J$ Cardiovasc Magn Reson 10, 40.

Youssef A, Ibrahim el SH, Korosoglou G, Abraham MR, Weiss RG \& Osman NF. (2008). Strain-encoding cardiovascular magnetic resonance for assessment of rightventricular regional function. J Cardiovasc Magn Reson 10, 33. 